\title{
The pain-relieving effects of lactoferrin on oxaliplatin-induced neuropathic pain
}

\author{
Takeshi FUJIMURA ${ }^{1)}$, Aiko IGUCHI ${ }^{1}$, Atsushi SATO2), Shinji KAGAYA ${ }^{3)}$, \\ Tatsuo HOSHINO ${ }^{3)}$ and Takashi TAKEUCHI ${ }^{1) *}$
1) Department of Veterinary Medicine, Faculty of Agriculture, Tottori University, 4-101 Koyama-Minami, Tottori 680-8553, Japan
${ }^{2)}$ School of Bioscience and Biotechnology, Tokyo University of Technology, 1404-1 Katakura, Hachioji, Tokyo 192-0982, Japan
${ }^{3)}$ NRL Pharma, Inc., \#203 KSP East, 3-2-1 Sakato, Takatsu-ku, Kawasaki, Kanagawa, 213-0012, Japan

\section{J. Vet. Med. Sci.}

82(11): 1648-1654, 2020

doi: 10.1292/jvms.20-0034

Received: 21 January 2020

Accepted: 14 September 2020

Advanced Epub:

25 September 2020

\begin{abstract}
Oxaliplatin (OXL) therapy often causes side effects including chronic peripheral neuropathy. We investigated the pain-relieving effects of recombinant human lactoferrin (rhLf) as well as a long-acting IgG-Fc fused rhLf (rhLf-Fc) on OXL-induced neuropathic pain. We used the hLf in this study, because the homology between mouse Lf and $h L f$ is higher than that of bovine Lf. In addition, rhLf-Fc is expected to enhance the analgesic effect due to the life extension effect in the body. We administered OXL ( $2 \mathrm{mg} / \mathrm{kg}$, i.v.) to mice twice weekly for 4 weeks. Phosphate buffered saline (PBS), rhLf (100 mg/kg, i.p.) or rhLf-Fc (100 mg/kg, i.p.) was administered once a week from day 15 to 32 . We also assessed the continuous infusion of same drugs ( $10 \mathrm{mg} / \mathrm{kg} /$ day) into the external jugular vein by using an osmotic pump. Both of rhLf and rhLf-Fc significantly reduced the hypersensitivity to mechanical stimulation when they were administered intraperitoneally. The continuous infusion of rhLf resulted in a more pronounced effect. Histopathological analysis of sciatic nerve showed that both rhLf and rhLf-Fc tended to reduce nerve fiber damage, but no significant difference was observed in nerve fiber cross-sectional area. Therefore, it was suggested that rhLf or rhLf-Fc injection could be an option for controlling neuropathic pain, which are side effects of OXL.
\end{abstract}

KEY WORDS: gait dysfunction, lactoferrin, neuropathic pain, oxaliplatin, side effects

Oxaliplatin (OXL) is a platinum-based anticancer drug and one of the key drugs used in the treatment of colorectal cancer [2]. Though the drug has good effects as an anticancer agent, it may be difficult to complete a full course of treatment because of its significant side effects. A peripheral neuropathy is frequently observed in OXL treated patients and is a dose-limiting toxicity [19] and a major cause of the discontinuation of treatment. Mechanical and cold allodynia observed due to OXL-induced chronic peripheral neuropathy is thought to be caused by cell damage that accompanies platinum accumulation. Acute or chronic peripheral neuropathy is often reversed with drug withdrawal [20]. However, if the withdrawal period is delayed, recovery may take a considerable amount of time. These symptoms significantly reduce the patient's quality of life (QOL) and there are currently no methods to prevent these side effects of OXL [2].

The physiological effects of lactoferrin (Lf) are diverse, and in recent years, it has been determined that Lf also has analgesic $[4,27]$ and anti-stress effects $[9,24]$. One of the mechanisms of analgesic effects of Lf is related with nitric oxide (NO) production and activation of endogenous $\mu$-opioid system [4, 27]. Tsuchiya et al. [27] reported that Lf has a potentiative effect on the morphine-induced analgesia through the following process: neuronal NO synthase (nNOS) activated selectively by Lf promotes NO production, and cGMP production stimulates by increased NO via guanylate cyclase (GC) induced potentiation for $\mu$-opioidreceptor-mediated analgesia.

Lf has been also reported to have a pain-relieving effect in a neuropathy model based on sciatic nerve ligation [15]. Prolonged, but not single, administration of bovine Lf produced antihyperalgesic and antiallodynic in neuropathic rats [15]. Lf has a short blood half-life, and some studies have failed to show the efficacy of Lf due to this short half-life. As a method of overcoming these problems, human lactoferrin (hLf)-human IgG Fc fusion protein with improved stability has been produced using IgG Fc fusion technology, which is a technology for improving blood stability of protein pharmaceuticals [22].

Aim of this study is to evaluate the pain relief effects of rhLf and rhLf-Fc in OXL-induced neuropathic pain model.

*Correspondence to: Takeuchi, T.: take3@tottori-u.ac.jp (Supplementary material: refer to PMC https://www.ncbi.nlm.nih.gov/pmc/journals/2350/) O2020 The Japanese Society of Veterinary Science 


\section{MATERIALS AND METHODS}

A total of 49 mice were used for this study. Seven mice were used in the control group, 21 mice were used in Experiment 1 (Exp. 1), and 21 mice were used in Experiment 2 (Exp.2). Five-week-old male ICR mice (Tokyo Experimental Animal, Tokyo, Japan) were used for these studies after a preliminary feeding trial and after a 1-week introductory period. Mice were housed in a laboratory animal room with room temperature $\left(22 \pm 2^{\circ} \mathrm{C}\right)$ and 12-hr light-dark cycle (light period 7:00-19:00). Mice were given ad libitum access to food (CE-2, Japan Clea, Tokyo, Japan) and water. The experiment was approved by the Tottori University Animal Experiment Committee. The approval number of the animal experiment in this study is "17-T-14".

Oxaliplatin (Elplat ${ }^{\circledR}$ ) was purchased from Yakult Honsha Co., Ltd. (Tokyo, Japan) and was diluted with 5\% (w/v) glucose solution (Terumo Co., Ltd., Tokyo, Japan) to create each concentration used in the studies. RhLf and rhLf-Fc were obtained from NRL Pharma, Inc. (Kawasaki, Japan) and diluted with PBS to create each study concentration. Using a similar methodology to a previous report [14], OXL (2 mg/kg) or 5\% (w/v) glucose solution was injected intravenously (i.v.) twice weekly for 4 weeks (at days $0,4,7,11,14,17,21,24$ and 28). Two weeks after the start of OXL administration, the mice served as a model for chronic injury of OXL-induced neuropathic pain in the studies described here.

In Exp.1, PBS ( $\mathrm{n}=7), \operatorname{rhLf}(100 \mathrm{mg} / \mathrm{kg}, \mathrm{n}=7)$, and $\mathrm{rhLf}-\mathrm{Fc}(100 \mathrm{mg} / \mathrm{kg}$ as $\mathrm{rhLf}$ conversion amount, $\mathrm{n}=7)$ were administered intraperitoneally (i.p.), once a week (immediately after the von Frey test at days 15, 22 and 29). The dose of drug was determined based on the previous report [4]. In addition, the experimental period was set at 4 weeks ( 2 weeks for drug administration) because the number of deaths increased with a certain number of times (days) when rhLf or rhLf-Fc were administered intraperitoneally in preliminary experiments. Survival rate in the experimental protocol using intraperitoneal administration was shown in the Supplementary Fig. 1.

In Exp.2, an osmotic pump (Alzet ${ }^{\circledR}$, Durect Corp., Cupertino, CA, USA) was implanted subcutaneously in the dorsal thoracic area at day 15 . PBS $(n=7), \operatorname{rhLf}(10 \mathrm{mg} / \mathrm{kg} /$ day, $n=7)$, and $\mathrm{rhLf}-\mathrm{Fc}(10 \mathrm{mg} / \mathrm{kg} / \mathrm{day}$ as $\mathrm{rhLf}$ conversion amount, $\mathrm{n}=7)$ were continuously infused into the external jugular vein from osmotic pump. We determined dose of drug with reference to previous report [5].

We evaluated the effects of rhLf and rhLf-Fc on mechanical allodynia using the von Frey test. This test was performed before the first drug administration (day 0) and 3 times per week for 4 weeks. Mice were placed in a clear plastic box $(12 \times 12 \times 12 \mathrm{~cm})$ with a wire mesh floor and allowed to habituate for $2 \mathrm{~min}$ prior to testing. We applied Filaments (TACTILE TEST AESTHESIO Semmes-Weinstein Von Frey Anesthesiometer; DanMic Global LLC, San Jose, CA, USA) in the range of 0.008-4 g bending force vertically to the footpad on right hindlimb of the mouse, with increasing stimulation. The intensity of the stimulus that resulted in the mouse raising the hindlimb 6 times for 10 stimuli was recorded as the $50 \%$ response threshold to the stimulus. We carried out the von Frey test as a blinded method.

Prior to evaluating motor function, the mouse was placed in a square box $(27.5 \times 27.5 \times 27.5 \mathrm{~cm})$ and allowed a 1 min acclimation time. Thereafter, the behavior of the mice was recorded for $5 \mathrm{~min}$ by video camera (web camera C270n, Logicool Co., Ltd., Tokyo, Japan), and the total moving distance and walking speed were measured and analyzed using SMART 3.0 software (Panlab, Harvard Apparatus, Barcelona, Spain). Walking behavior (traveling distance, mean speed, fast moving $(>5.0 \mathrm{~cm} / \mathrm{sec})$ and slow moving $(<5.0 \mathrm{~cm} / \mathrm{sec})$ was recorded once a week. Data is expressed as percentage based on Day 14 (chronic peripheral neuropathy).

After the completion of the Exp.1, euthanasia was performed through isoflurane overdose, and the sciatic nerve was collected. The sciatic nerve was fixed in 10\% formalin, and then embedded in paraffin using standard techniques. Slices were stained with Hematoxylin-Eosin and then examined using a digital microscope BZ-9000 (KEYENCE Corp., Osaka, Japan). The axon area of each nerve fiber of the sciatic nerve was calculated by using the image analysis software BZ-X Analyzer (KEYENCE Corp.) from approximately 1,500 to 3,000 axons per rat. Values are expressed as the mean \pm SE means of four to six animals in each group.

Statistical analyses were carried out using repeated one-way ANOVA and Tukey's multiple comparison test as a post-test using software (Prism4, GraphPad software, San Diego, CA, USA) to evaluate the von Frey latency from day 0 to 13 and day 18 to 32 in the Exp.1. Repeated one-way ANOVA was also carried out to evaluate the von Frey latency from day 16 to 30 in the Exp.2. Kruskal-Wallis test and Steel test as a post-test were carried out using software (BellCurve, Tokyo, Japan) for comparison mean axon area of sciatic nerve fiber in the Exp.1 and walking behavior in the Exp.2. In all cases, the significance level was set at $P<0.05$.

\section{RESULTS}

The results of the von Frey test from day 0 to day 13 (chronic injury model preparation period) in Exp. 1 are shown in Fig. 1. In the Glucose + PBS group, there was no reduction in the 50\% response threshold from day 0 to 13 . In contrast, all three groups administered OXL showed a gradual decrease of the 50\% response threshold until day 13 . There was a significant difference between the Glucose + PBS and OXL administered groups on day 13.

After the rhLf or rhLf-Fc administration in the Exp.1, 50\% response thresholds in OXL + rhLf groups and OXL + rhLf-Fc groups were significantly higher when compared with the OXL + PBS group at the day 32 (Fig. 1).

Figure 2 shows photographs of tissue cross sections of the sciatic nerve in Exp.1. Particular atrophy of myelinated fibers was confirmed in OXL + PBS group. Degenerating fibers, indicative of a slight axonopathy, were observed in OXL + PBS group. In contrast, the atrophies of the myelinated fibers were reduced in both OXL + rhLf and OXL + rhLf-Fc groups. 


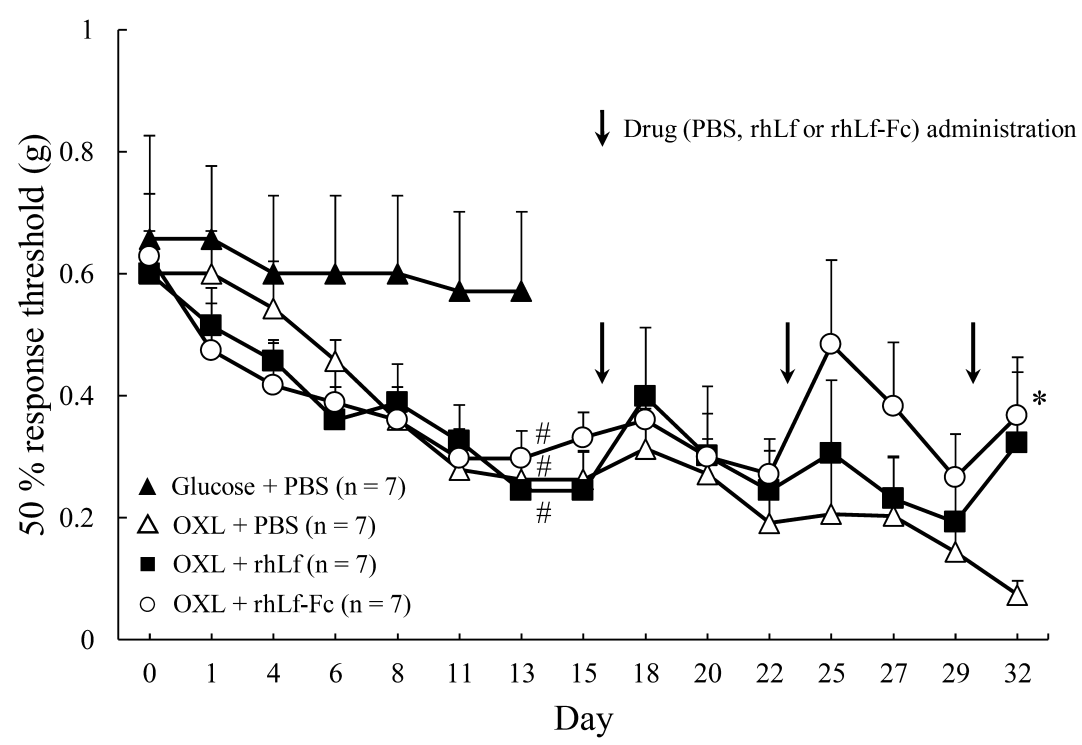

Fig. 1. Changes in the 50\% response threshold to mechanical stimulation in oxaliplatin administration in Experiment 1. (Note: From day 0 to 13 , only Oxaliplatin (OXL) was administered. Phosphate buffered saline (PBS), recombinant human lactoferrin (rhLF) and recombinant human lactoferrin Fc fusion (rhLF-Fc) were administered intraperitoneally once a week, immediately after the von Frey test at days 15, 22 and 29. PBS, rhLf or rhLf-Fc administration is indicated by Arrow mark. Response threshold is expressed as mean \pm SE. Repeated one-way ANOVA was performed on data from day 0 to 13 that only OXL administration period. In the same way, repeated one-way ANOVA was done on data from 18 to 32 to evaluate the effects of drug (PBS, rhLf or rhLf-Fc) administration. \#; $P<0.05$ vs. Glucose + PBS group. *; $P<0.05$ vs. OXL + PBS group.
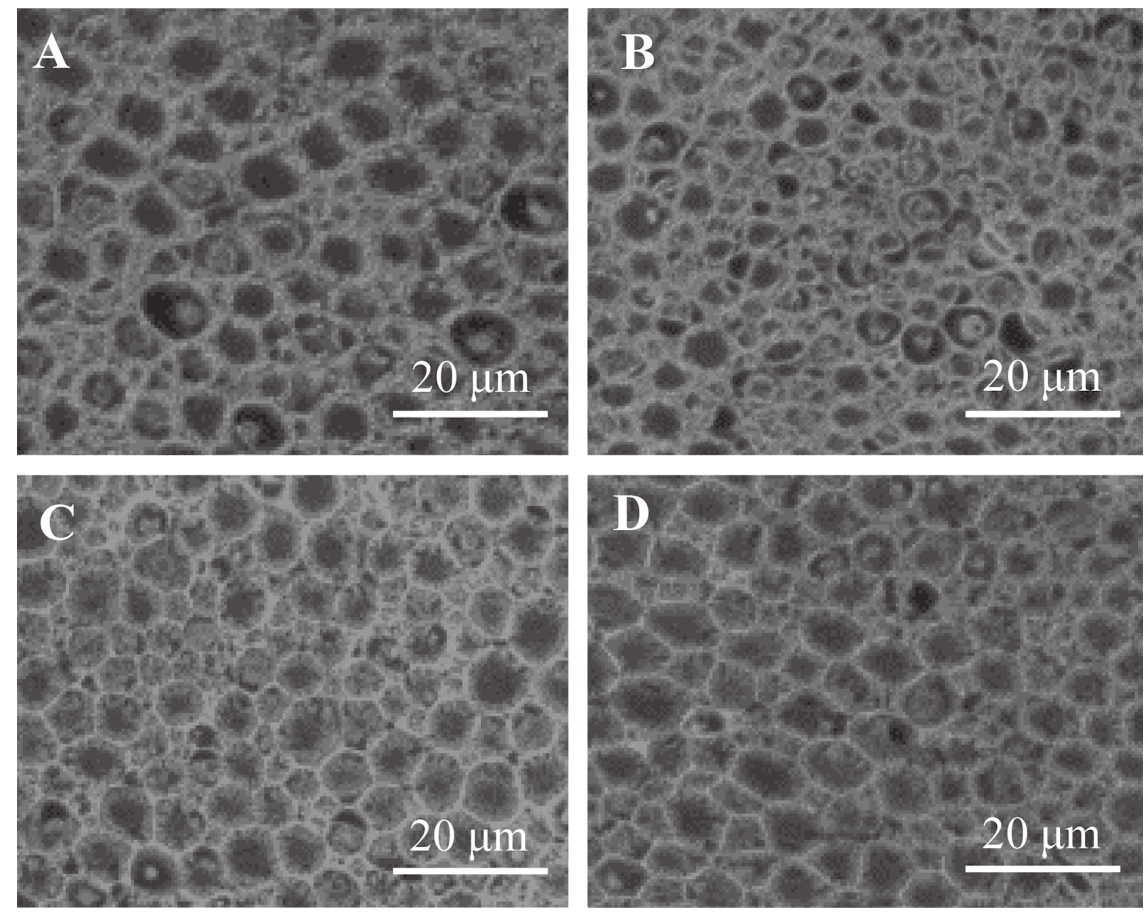

Fig. 2. High-magnification image of nerve fibers in sciatic nerve in Experiment 1 (Exp.1). Sciatic nerve was collected on day 32 in the Exp.1. A; Glucose + Phosphate buffered saline (PBS) group, B; Oxaliplatin (OXL) + PBS group, C; OXL + recombinant human lactoferrin (rhLF) group, D; OXL + recombinant human lactoferrin Fc fusion (rhLF-Fc) group. The samples were stained with Hematoxylin-Eosin. Scale bar $=20 \mu \mathrm{m}$. In Image B, atrophy and loss of nerve fibers was observed.

In Fig. 3, the axons of the sciatic nerve were shown as green color to calculate the axon area. Mean axon area of each nerve fiber was $26.2 \pm 1.0 \mu \mathrm{m}^{2}, 24.6 \pm 0.7 \mu \mathrm{m}^{2}, 26.2 \pm 0.4 \mu \mathrm{m}^{2}$ and $25.8 \pm 1.6 \mu \mathrm{m}^{2}$ in the Glucose + PBS group, OXL + PBS group, OXL + rhLf group and OXL + rhLf-Fc group, respectively. Although the axon area in the OXL + PBS was seemed to be decrease, there was no significant difference between the Glucose + PBS and OXL + PBS group. 


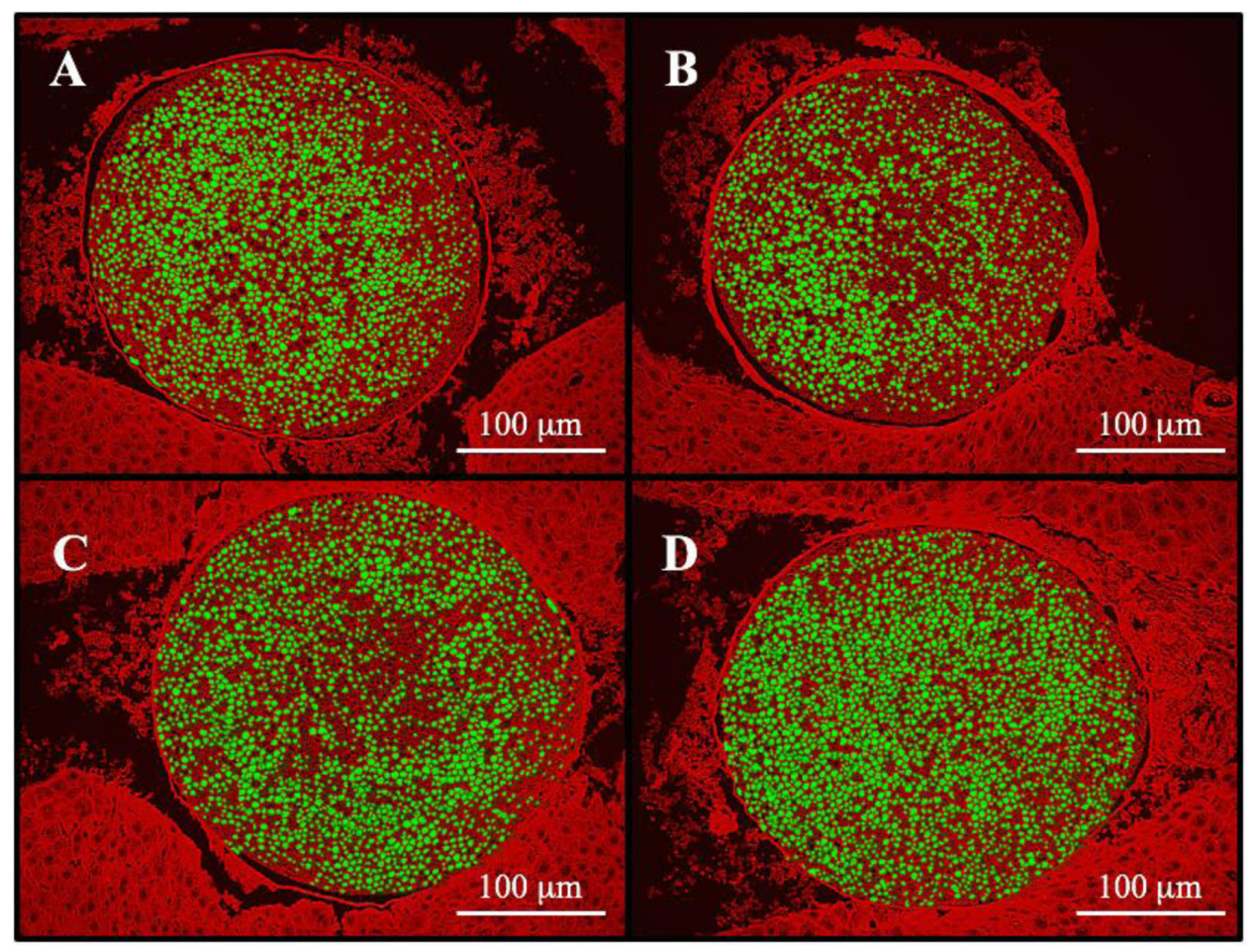

Fig. 3. Comparison of axon area in sciatic nerve tissue in Experiment 1 (Exp.1). Sciatic nerve was collected on day 32 in the Exp.1. A; Glucose + Phosphate buffered saline (PBS) group, B; Oxaliplatin (OXL) + PBS group, C; OXL + recombinant human lactoferrin (rhLF) group, D; $\mathrm{OXL}+$ recombinant human lactoferrin Fc fusion (rhLF-Fc) group. Each nerve fiber is indicated in green color. Scale bar=100 $\mu \mathrm{m}$. B; Atrophy of nerve fibers is observed in the OXL + PBS group, and the nerve fiber bundles are more sparse compared with the control group. C, D; OXL + rhLF group and OXL + rhLF-Fc group, nerve fiber bundles are more densely than that in the OXL + PBS group.

The results of von Frey test in Exp.2 are shown in Fig. 4. Osmotic pump was implanted at the day 15, thereafter PBS, rhLf or rhLf-Fc was continuously administered into the external jugular vein. Remarkable hypersensitivity to mechanical stimulation was confirmed in the OXL + PBS group up to the day 30. In contrast, the hypersensitivity was significantly attenuated by the continuous intravenous infusion of rhLf or rhLf-Fc.

The evaluation of walking behavior in Exp.2 is shown in Fig. 5. Although there was a decrease in the total traveled distance and the rate of fast moving $(>5.0 \mathrm{~cm} / \mathrm{sec})$ in the OXL + PBS group, they were maintained in OXL + rhLf and OXL + rhLf-Fc groups. The trends seen in these results, although not significantly different, suggest that rhLf or rhLf-Fc treatment support the motor function. There was no significant difference between groups in the rates of slow moving $(<5.0 \mathrm{~cm} / \mathrm{sec})$ and average walking speeds.

\section{DISCUSSION}

In this study, rhLf and rhLf-Fc reduced OXL-induced neuropathic pain. This will contribute both to the problems of treatment cessation that often occur in the treatment of anti-cancer drugs with OXL and to improving the QOL of treated patients. Lf itself is a drug that does not have serious side effects, and it could be one of the tools to overcome the problem of the side effects of OXL. The mechanical allodynia observed in chronic peripheral neuropathy due to OXL is thought to be caused by damage to the cell body induced by platinum compounds accumulated in nerve cells [20]. In addition, it has been shown that phosphorylation of $\mathrm{Ca}^{2+} /$ calmodulin dependent protein kinase II (CaMKII) in the spinal cord was significantly increased by repeated administration of OXL, which was suppressed by NMDA receptor NR2B inhibitor [23]. There have been several reports of drug effects on OXLinduced peripheral neuropathy. NMDA receptor antagonists Memantine, NR2B inhibitory ifenprodil and calmodulin inhibitory trifluoperazine, which inhibits CaMKII activity, temporarily improve mechanical allodynia [12, 23]. Furthermore, amitriptyline suppresses NR2B expression to prevent mechanical allodynia [17]. Neurotropin did not suppress OXL-induced DRG cell damage, but was found to prevent axon degeneration of the sciatic nerve and moderately suppress the development of mechanical allodynia [10]. However, these drugs have side effects as mentioned above. It has also been reported that Lf is effective in preventing colorectal cancer [7, 11, 13], and it is considered that Lf may have a synergistic therapeutic / preventive effect on colorectal cancer when used in combination with OXL. The combination of rhLf and rhLf-Fc with OXL has advantages over the combination with other drugs.

The oral administration of bLf is generally used in humans. In addition, based on the report [25] that when bLf was orally 
administered at $5.5 \pm 1.0 \mathrm{mg} / \mathrm{kg}$ in newborn calves, the peak blood concentration after $8 \mathrm{hr}$ was $2.4 \mu \mathrm{g} / \mathrm{ml}$, the absorption rate of bLf after oral administration is expected to be approximately $0.05 \%$. By using hLf, direct intravascular administration is possible, and more direct effects of $\mathrm{Lf}$ (securing the blood concentration) can be expected in human. We chose intraperitoneal or intravenous administration as a comparison of different routes of oral administration.

The Fc was used to enhance the stability of hLf in blood [21, 22], to increase the overall effect. Although hLf is metabolized in the liver, it is thought to be modified by Fc to avoid catabolism after being taken into the liver. However, rhLf significantly reduced the sensitivity to physical stimulus, but the effect of rhLf-Fc was less than that of rhLf in the present study. Pain-relieving action of hLf may be weakened due to factors such as an increase in the molecular weight of bound Fc fusion protein, steric hindrance caused by Fc fusion and a decrease in metabolic activity.

RhLf and rhLf-Fc alleviated sciatic nerve atrophy caused by OXL in the histopathological study (Fig. 2). It has been reported that $\mathrm{Lf}$ alleviates neuronal oxidative damage by antioxidant action $[8,18]$. Together with these previous reports, it is expected that oxidative damage due to OXL might be reduced by treatment with rhLf or rhLf-Fc, and pain was alleviated. Oxidative damage is maximized at 48 to $72 \mathrm{hr}$ after OXL administration [1]. When a high dose or cumulative dose of OXL exceeded the threshold, irreversible neuropathy developed [19]. In this study, the progression of the hypersensitivity response was observed with twice weekly administration of OXL. After multiple administrations, the hypersensitivity response gradually progressed until day 14 .

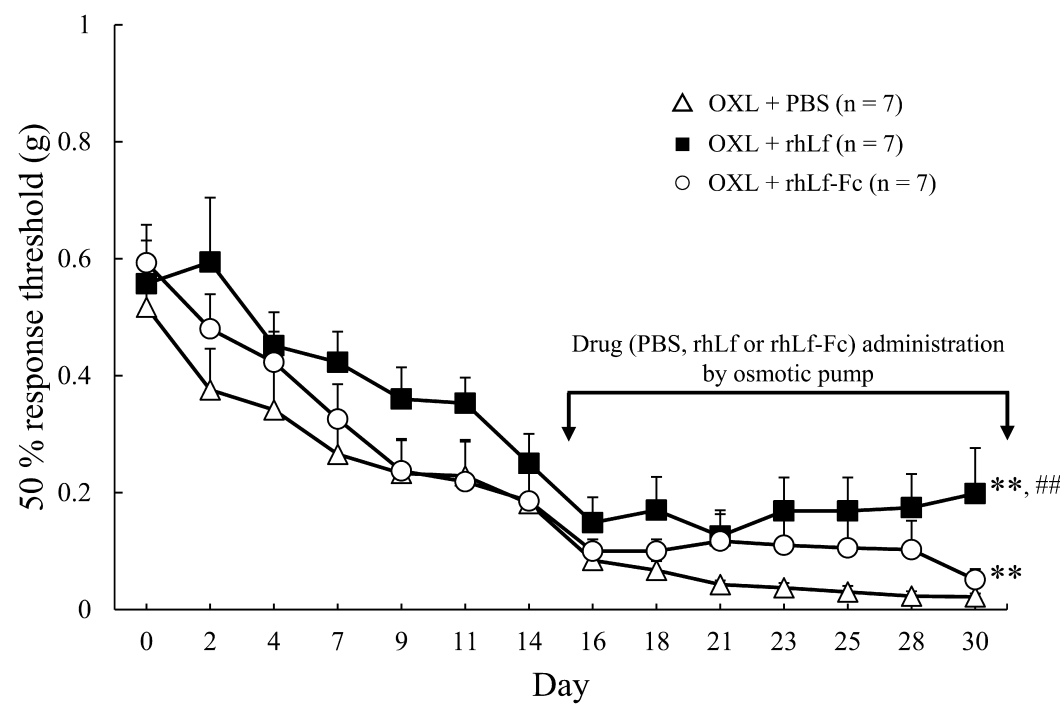

Fig. 4. Changes in the 50\% response threshold to mechanical stimulation in Experiment 2. Phosphate buffered saline (PBS), recombinant human lactoferrin (rhLF) and recombinant human lactoferrin Fc fusion (rhLF-Fc) were continuously infused into the external jugular vein using an osmotic pump. The response threshold is expressed as mean \pm SE. Repeated one-way ANOVA was performed on data from day 15 to 30 , the drug (PBS, rhLf or rhLf-Fc) administration period. **; $P<0.01$ vs. Oxaliplatin (OXL) + PBS group, \#\#; $P<0.01$ vs. OXL + rhLf-Fc group, respectively.
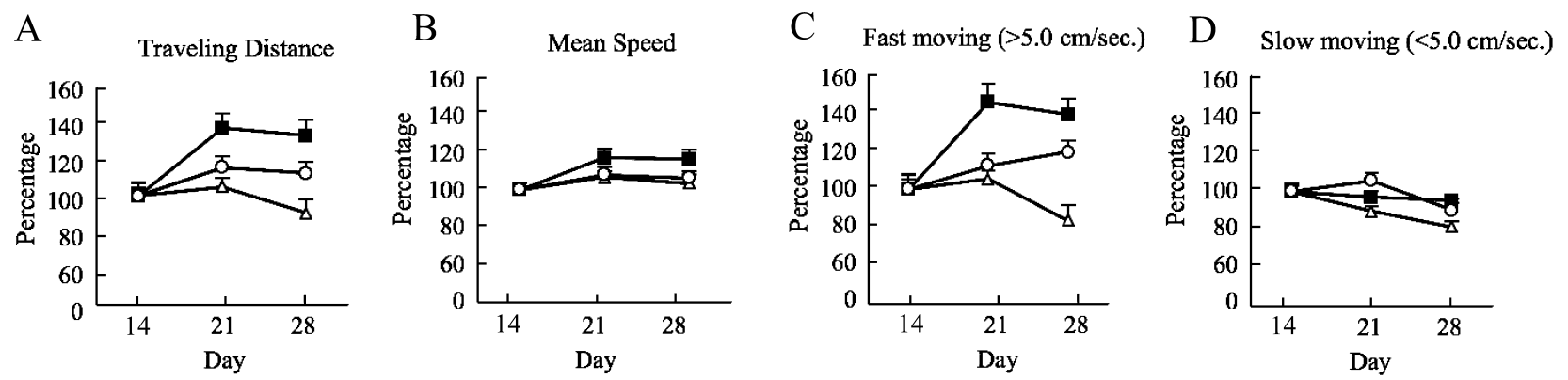

Fig. 5. Changes in walking behavior of each group in Experiment 2. $\Delta$; Oxaliplatin (OXL) + Phosphate buffered saline (PBS), $\mathbf{m}$; OXL + recombinant human lactoferrin (rhLf), O; OXL + recombinant human lactoferrin Fc fusion (rhLf-Fc). A; Traveling Distance, B; Mean Speed, C; Fast moving $(>5.0 \mathrm{~cm} / \mathrm{sec})$, D; Slow moving $(<5.0 \mathrm{~cm} / \mathrm{sec})$ The vertical axis of the graph shows the score ratio, with the score at chronic peripheral neuropathy (day 14) as $100 \%$. Ratios are expressed as mean \pm SE. The horizontal axis of the graph indicates the second week of OXL administration (day 14), the first week of drug administration (day 21), and the second week of drug administration (day 28). Data were statistically analyzed using Kruskal-Wallis test. 
Thereafter, the hypersensitivity response continued in the OXL+PBS group (control group) until day 30 . This protocol reliably caused neuropathic pain and the development of chronic pain without transient recovery, as has been described in previous reports $[16,26]$. In this study, since OXL was administered twice a week, it is believed that neurodegeneration was associated with the cumulative effects of repeated administration. RhLf and rhLf-Fc appear to have mitigated this deterioration; further studies should explore the time course of these effects.

It was also reported that hyperalgesia in a model of strangulated nerve injury was suppressed by NMDA receptor antagonists [12]. In the sciatic nerve ligation model, enhancement of excitatory transmission from primary sensory neurons to secondary sensory neurons in dorsal root ganglion (DRG) is considered to be the main mechanism of mechanical allodynia remarks [3]. Since Lf also has a significant analgesic effect on the sciatic nerve ligation model, it has been pointed out that the point of action of Lf may be DRG [15]. The involvement of DRG in the mechanism of action of Lf in the sciatic nerve ligation model has been reported, so it is possible that the Lf action point for OXL-induced neuropathic pain may be the DRG. In this study, we have not yet verified the gene expression in DRG, and we think that it should be clarified in the future. However, as described above, the OXL-induced neuropathy is a combination of multiple mechanisms such as oxidative damage and CaMKII phosphorylation, so it is necessary to clarify the effect of Lf on these mechanisms.

Antibodies may be produced when a heterologous protein is administered in vivo, and in particular, high levels of antibody production have been reported by subcutaneous administration [6]. The passive cutaneous anaphylaxis antibody titers in the subcutaneous administration group were in the range of 1,000 to 10,000 [6]. This is reason why in humans lactoferrin supplements have been limited to oral ingestion. In this study, assuming human applicability, rhLf and rhLf-Fc were administered intraperitoneally and intravenously. Administration of rhLf to mice is concerned about antibody production in the body, but in this study, as one of the solutions to this problem, we expect that Fc coexpressed rhLf-Fc will reduce the antigenicity. However, longterm Lf administration caused shock-like symptoms in many mice and some died. In this study, we set the endpoint at 1 month and administered the optimal amount of Lf in that condition. If further long-term administration and evaluation are to be continued, it is necessary to reconsider the dose. Moreover, it is considered that antigenicity is unlikely to be expressed when Lf is administered at very small speed by an osmotic pump because the blood concentration will not rise sharply. As a long-term administration protocol, it is expected that a small amount of intravascular administration will be more applicable.

In conclusion, rhLf and rhLf-Fc were shown to alleviate neuropathic pain caused by OXL-induced neuropathy. RhLf-Fc showed effects that were similar to or better than rhLf when administered intraperitoneally. However, rhLf was significantly more effective when continuously administered with low dose infusion via an osmotic pump. For clinical applications, continuous administration may be desirable, but the use of rhLf modified with IgG may not enhance these effects. Further studies are needed to clarify the factors that maximize the pain-relieving effect of hLf for OXL-induced neuropathic pain.

\section{REFERENCES}

1. Candelario-Jalil, E., Mhadu, N. H., Al-Dalain, S. M., Martínez, G. and León, O. S. 2001. Time course of oxidative damage in different brain regions following transient cerebral ischemia in gerbils. Neurosci. Res. 41: 233-241. [Medline] [CrossRef]

2. de Gramont, A., Figer, A., Seymour, M., Homerin, M., Hmissi, A., Cassidy, J., Boni, C., Cortes-Funes, H., Cervantes, A., Freyer, G., Papamichael, D., Le Bail, N., Louvet, C., Hendler, D., de Braud, F., Wilson, C., Morvan, F. and Bonetti, A. 2000. Leucovorin and fluorouracil with or without oxaliplatin as first-line treatment in advanced colorectal cancer. J. Clin. Oncol. 18: 2938-2947. [Medline] [CrossRef]

3. Dworkin, R. H., O’Connor, A. B., Backonja, M., Farrar, J. T., Finnerup, N. B., Jensen, T. S., Kalso, E. A., Loeser, J. D., Miaskowski, C., Nurmikko, T. J., Portenoy, R. K., Rice, A. S., Stacey, B. R., Treede, R. D., Turk, D. C. and Wallace, M. S. 2007. Pharmacologic management of neuropathic pain: evidence-based recommendations. Pain 132: 237-251. [Medline] [CrossRef]

4. Hayashida, K., Takeuchi, T., Shimizu, H., Ando, K. and Harada, E. 2003. Lactoferrin enhances opioid-mediated analgesia via nitric oxide in the rat spinal cord. Am. J. Physiol. Regul. Integr. Comp. Physiol. 285: R306-R312. [Medline] [CrossRef]

5. Hu, K., Shi, Y., Jiang, W., Han, J., Huang, S. and Jiang, X. 2011. Lactoferrin conjugated PEG-PLGA nanoparticles for brain delivery: preparation, characterization and efficacy in Parkinson's disease. Int. J. Pharm. 415: 273-283. [Medline] [CrossRef]

6. Ishikado, A., Imanaka, H., Takeuchi, T., Harada, E. and Makino, T. 2005. Liposomalization of lactoferrin enhanced it's anti-inflammatory effects via oral administration. Biol. Pharm. Bull. 28: 1717-1721. [Medline] [CrossRef]

7. Jiang, R. and Lönnerdal, B. 2017. Bovine lactoferrin and lactoferricin exert antitumor activities on human colorectal cancer cells (HT-29) by activating various signaling pathways. Biochem. Cell Biol. 95: 99-109. [Medline] [CrossRef]

8. Joubran, Y., Mackie, A. and Lesmes, U. 2013. Impact of the Maillard reaction on the antioxidant capacity of bovine lactoferrin. Food Chem. 141: 3796-3802. [Medline] [CrossRef]

9. Kamemori, N., Takeuchi, T., Hayashida, K. and Harada, E. 2004. Suppressive effects of milk-derived lactoferrin on psychological stress in adult rats. Brain Res. 1029: 34-40. [Medline] [CrossRef]

10. Kawashiri, T., Egashira, N., Watanabe, H., Ikegami, Y., Hirakawa, S., Mihara, Y., Yano, T., Ikesue, H. and Oishi, R. 2011. Prevention of oxaliplatininduced mechanical allodynia and neurodegeneration by neurotropin in the rat model. Eur. J. Pain 15: 344-350. [Medline] [CrossRef]

11. Kozu, T., Iinuma, G., Ohashi, Y., Saito, Y., Akasu, T., Saito, D., Alexander, D. B., Iigo, M., Kakizoe, T. and Tsuda, H. 2009. Effect of orally administered bovine lactoferrin on the growth of adenomatous colorectal polyps in a randomized, placebo-controlled clinical trial. Cancer Prev. Res. (Phila.) 2: 975-983. [Medline] [CrossRef]

12. Mihara, Y., Egashira, N., Sada, H., Kawashiri, T., Ushio, S., Yano, T., Ikesue, H. and Oishi, R. 2011. Involvement of spinal NR2B-containing NMDA receptors in oxaliplatin-induced mechanical allodynia in rats. Mol. Pain 7: 8. [Medline] [CrossRef]

13. Moastafa, T. M., El-Sissy, Ael-D., El-Saeed, G. K. and Koura, M. S. 2014. Study on the therapeutic benefit on lactoferrin in patients with colorectal cancer receiving chemotherapy. Int. Sch. Res. Notices. 10.1155/2014/184278. [Medline]

14. Ohsawa, M., Otake, S., Murakami, T., Yamamoto, S., Makino, T. and Ono, H. 2014. Gabapentin prevents oxaliplatin-induced mechanical hyperalgesia in mice. J. Pharmacol. Sci. 125: 292-299. [Medline] [CrossRef] 
15. Onal, A., Kayalioğlu, G., Parlar, A., Keser, A. and Ulker, S. 2010. Effect of prolonged administration of bovine lactoferrin in neuropathic pain: involvement of opioid receptors, nitric oxide and TNF-alpha. Life Sci. 86: 251-259. [Medline] [CrossRef]

16. Park, J. H., Chae, J., Roh, K., Kil, E. J., Lee, M., Auh, C. K., Lee, M. A., Yeom, C. H. and Lee, S. 2015. Oxaliplatin-induced peripheral neuropathy via TRPA1 stimulation in mice dorsal root ganglion is correlated with aluminum accumulation. PLoS One 10: e124875. [Medline] [CrossRef]

17. Sada, H., Egashira, N., Ushio, S., Kawashiri, T., Shirahama, M. and Oishi, R. 2012. Repeated administration of amitriptyline reduces oxaliplatininduced mechanical allodynia in rats. J. Pharmacol. Sci. 118: 547-551. [Medline] [CrossRef]

18. Safaeian, L., Javanmard, S. H., Mollanoori, Y. and Dana, N. 2015. Cytoprotective and antioxidant effects of human lactoferrin against $\mathrm{H}_{2} \mathrm{O}_{2}$-induced oxidative stress in human umbilical vein endothelial cells. Adv. Biomed. Res. 4: 188. 10.4103/2277-9175.164010. [Medline]

19. Saif, M. W. and Reardon, J. 2005. Management of oxaliplatin-induced peripheral neuropathy. Ther. Clin. Risk Manag. 1: 249-258. [Medline]

20. Sakurai, M., Egashira, N., Kawashiri, T., Yano, T., Ikesue, H. and Oishi, R. 2009. Oxaliplatin-induced neuropathy in the rat: involvement of oxalate in cold hyperalgesia but not mechanical allodynia. Pain 147: 165-174. [Medline] [CrossRef]

21. Shiga, Y., Murata, D., Sugimoto, A., Oshima, Y., Tada, M., Ishii-Watabe, A., Imai, K., Tomii, K., Takeuchi, T., Kagaya, S. and Sato, A. 2017. Hingedeficient IgG1 Fc fusion: application to human lactoferrin. Mol. Pharm. 14: 3025-3035. [Medline] [CrossRef]

22. Shiga, Y., Oshima, Y., Kojima, Y., Sugimoto, A., Tamaki, N., Murata, D., Takeuchi, T. and Sato, A. 2015. Recombinant human lactoferrin-Fc fusion with an improved plasma half-life. Eur. J. Pharm. Sci. 67: 136-143. [Medline] [CrossRef]

23. Shirahama, M., Ushio, S., Egashira, N., Yamamoto, S., Sada, H., Masuguchi, K., Kawashiri, T. and Oishi, R. 2012. Inhibition of $\mathrm{Ca}^{2+} / \mathrm{calmodulin}^{-}$ dependent protein kinase II reverses oxaliplatin-induced mechanical allodynia in rats. Mol. Pain 8: 26. [Medline] [CrossRef]

24. Takeuchi, T., Hayashida, K., Inagaki, H., Kuwahara, M., Tsubone, H. and Harada, E. 2003. Opioid mediated suppressive effect of milk-derived lactoferrin on distress induced by maternal separation in rat pups. Brain Res. 979: 216-224. [Medline] [CrossRef]

25. Talukder, M. J., Takeuchi, T. and Harada, E. 2002. Transport of colostral macromolecules into the cerebrospinal fluid via plasma in newborn calves. J. Dairy Sci. 85: 514-524. [Medline] [CrossRef]

26. Tsubaki, M., Takeda, T., Tani, T., Shimaoka, H., Suzuyama, N., Sakamoto, K., Fujita, A., Ogawa, N., Itoh, T., Imano, M., Funakami, Y., Ichida, S., Satou, T. and Nishida, S. 2015. PKC/MEK inhibitors suppress oxaliplatin-induced neuropathy and potentiate the antitumor effects. Int. J. Cancer 137: 243-250. [Medline] [CrossRef]

27. Tsuchiya, T., Takeuchi, T., Hayashida, K., Shimizu, H., Ando, K. and Harada, E. 2006. Milk-derived lactoferrin may block tolerance to morphine analgesia. Brain Res. 1068: 102-108. [Medline] [CrossRef] 